\title{
RINGS WITH ASSOCIATORS IN THE COMMUTATIVE CENTER
}

\author{
ERWIN KLEINFELD
}

\author{
(Communicated by Donald S. Passman)
}

\begin{abstract}
Thedy has introduced the subject of rings which satisfy the identity

$(R,(R, R, R))=0$,

where the commutator is defined by $(a, b)=a b-b a$, and the associator is defined by $(a, b, c)=a b \cdot c-a \cdot b c$ and which satisfy one additional identity such as $(x, x, x)=0$. Assuming characteristic $\neq 2$ and simplicity, Thedy's result is that such a ring $R$ must be either commutative or associative. Thedy also showed that simplicity could not be relaxed to prime by presenting some examples which are neither associative nor commutative. We show here that the additional identity assumed by Thedy is in fact unnecessary for we show that if $R$ is simple, characteristic $\neq 2,3$ and satisfies (I) then $R$ must be either commutative or associative.
\end{abstract}

1. Introduction. Thedy has introduced the subject of rings which satisfy the identity

$$
(R,(R, R, R))=0
$$

where the commutator is defined by $(a, b)=a b-b a$, and the associator is defined by $(a, b, c)=a b \cdot c-a \cdot b c$ and which satisfy one additional identity such as $(x, x, x)=0$. Assuming characteristic $\neq 2$ and simplicity, Thedy's result is that such a ring $R$ must be either commutative or associative. Thedy also showed that simplicity could not be relaxed to prime by presenting some examples which are neither associative nor commutative. We show here that the additional identity assumed by Thedy is in fact unnecessary for we show that if $R$ is simple, characteristic $\neq 2,3$ and satisfies (I) then $R$ must be either commutative or associative.

2. Main result. In every ring the so-called semi-Jacobi identity

$$
(x y, z)=x(y, z)+(x, z) y+(x, y, z)+(z, x, y)-(x, z, y) \text {, }
$$

is known to hold. One need only expand the right-hand side of (1) and cancel terms to verify this. Next define $U=\{u \in R \mid(u, R)=0\}$. $U$ is often called the commutative center of $R$. Then define $V=\{v \in U \mid v R \subset U\}$.

LEMMA. $V$ is an ideal on $R$.

PROOF. Since $V \subset U$ it suffices to show $V$ is a right ideal. Let $v \in V$. Then for all $r, s \in R, v r \in U$ follows from the definition of $V$. Since (I) implies $(v, r, s) \in U$ and $v r \cdot s=(v, r, s)+v \cdot r s \in U$, it follows that $v r \in V$. This concludes the proof of the lemma.

Received by the editors August 19, 1987.

1980 Mathematics Subject Classification (1985 Revision). Primary 17A30. 
Assume henceforth that $R$ is not commutative. Hence $V \neq R$. Since $R$ is simple then because of the lemma we are reduced to the case where $V=0$. In every ring we have the so-called Teichmuller identity.

$$
(w x, y, z)-(w, x y, z)+(w, x, y z)=w(x, y, z)+(w, x, y) z
$$

The proof follows from expanding each side and using the definition of the associator. Commuting each term in (2) with $r$ and using (I) results in $(r, w(x, y, z))+$ $(r,(w, x, y) z)=0$, so that $(r, w(x, y, z))=-(r,(w, x, y) z)=-(r, z(w, x, y))$ using (I). By permuting cyclically $(w z y x)$ thus

$$
(r, w(x, y, z))=-(r, z(w, x, y))=(r, y(z, w, x))=-(r, x(y, z, w)) .
$$

Suppose that $a$ is an arbitrary associator of $R$. Let $y=x$ and $z=a$ in (1) and use (I). Thus $\left(x^{2}, a\right)=x(x, a)+(x, a) x+(x, x, a)+(a, x, x)-(x, a, x)$, so that

$$
(x, x, a)+(a, x, x)-(x, a, x)=0 .
$$

Multiplying the terms in (4) on the left by $x$ and commuting with $z$ we obtain

$$
(z, x(x, x, a)+x(a, x, x)-x(x, a, x))=0 .
$$

Using (3) on (5) we discover that $-(z, a(x, x, x))-(z, a(x, x, x))-(z, a(x, x, x))=0$, or $-3(z, a(x, x, x))=0$. Thus $(z, a(x, x, x))=0$. Since $a$ is an arbitrary associator we may replace $a$ by $(b, c, d)$, so that

$$
(z,(b, c, d)(x, x, x))=0 .
$$

Then using (I) we obtain from (6) that

$$
(z,(x, x, x)(b, c, d))=0 .
$$

Applying (3) to (7) we obtain

$$
(z, b(c, d,(x, x, x)))=0=(z, c(d,(x, x, x), b))=(z, d((x, x, x), b, c)) .
$$

But (8) and (I) prove that $(c, d,(x, x, x)) \in V,(d,(x, x, x), b) \in V$ and $((x, x, x), b, c)$ $\in V$. Since $V=0,(x, x, x)$ must be in the nucleus of $R$. This combined with (I) proves that $(x, x, x)$ is in the center of $R$. Next apply (3) to $(z, x(x, x, x))$. Thus $(z, x(x, x, x))=-(z, x(x, x, x))$. This leads to $2(z, x(x, x, x))=0$, so that

$$
(z, x(x, x, x))=0 \text {. }
$$

Expanding $(x(x, x, x), z)=0$ by using the semi-Jacobi identity we have $0=x((x, x, x), z)+(x, z)(x, x, x)+(x,(x, x, x), z)+(z, x,(x, x, x))-(x, z,(x, x, x))$.

However $(x, x, x)$ is in the center. Thus only one term survives and we obtain

$$
(x, z)(x, x, x)=0 \text {. }
$$

Now let $z=-x^{2}$ in (10). Since $-\left(x, x^{2}\right)=x^{2} x-x x^{2}=(x, x, x)$ we obtain

$$
(x, x, x)^{2}=0 \text {. }
$$

Let $q=(x, x, x)$. Then $q$ is a center element and now (11) implies $q^{2}=0$. From this it is well known that the ideal generated by $q$ also squares to zero. If that ideal were all of $R$, then $R$ would be both commutative and associative. We have already assumed that $R$ is not commutative. Thus the ideal generated by $q$ must be zero, which in turn implies $q=0$. So $q=(x, x, x)=0$. This is the additional identity that Thedy used in [2]. Thus we have established the following result. 
MAIN THEOREM. A simple ring of characteristic $\neq 2,3$ which satisfies (I) must be associative or commutative.

Of course commutative rings satisfy (I) and associative rings satisfy (I). Simplicity can not be relaxed to prime in the main theorem without giving up the conclusion because of Thedy's examples in [2]. Rings with associators in the nucleus were studied in [1] and one needs only assume semiprime to show that such rings are always associative [1].

\section{REFERENCES}

1. Erwin Kleinfeld, $A$ class of rings which are very nearly associative, Amer. Math. Monthly 93 (1986), 720-722.

2. Armin Thedy, On rings satisfying $((a, b, c), d)=0$, Proc. Amer. Math. Soc. 29 (1971), 213218.

Department of Mathematics, University of IOWA, IOWA City, IOWA 52242 